\title{
OBSTRUCTIONS IN SHAPE THEORY
}

\author{
BY TIM PORTER \\ Communicated by Glen Bredon, April 3, 1973
}

1. Introduction. The classical theory of obstructions (see, for instance, Spanier [5]) applied only to the case where all the spaces concerned have the homotopy type of a CW-complex, however the work on shape theory by the group of Polish topologists around Borsuk and, more recently, by Mardešić and Segal (see [2]) has indicated that it is possible to adapt classical homotopy theory to give useful information on, for instance, general compact Hausdorff spaces. At the same time, the author has, independently, developed a rudimentary homotopy theory [3] which complements the classical Čech homology and cohomology theories. This theory has close connections with Borsuk's theory, but has more of the flavour of Artin and Mazur's work on the étale homotopy theory in algebraic geometry. (The connections with shape theory are explored in [4].)

The attack of both these approaches has been restricted, albeit successfully, to the relatively simple parts of homotopy theory; it therefore seemed natural to attack one of the deeper areas from a similar viewpoint as a next step in the development of the theories. This note announces the initial results of my attempt to study the extension and lifting problems from this point of view. The details will, I hope, be published later.

2. A cohomology theory on a category of diagrams. Although the methods of both shape theory and Čech homotopy use inverse systems of polyhedra, it is simpler to develop our obstruction theory in a category of diagrams of pointed $\mathrm{CW}$-complexes and to translate to the more general case later.

The category of pointed CW-complexes and cellular maps is denoted by $\mathrm{CW}_{*}$ and $\Sigma$ will denote the ordered linear category of positive integers where there is a unique map from $m$ to $n$ as soon as $m \geqq n$.

Suppose $F: \Sigma \rightarrow A b$ is a diagram of abelian groups and $C_{*}: \Sigma \rightarrow C(A b)$ a diagram of chain complexes of abelian groups. A collection

$$
f=\left\{f(\sigma): C_{n}(\sigma) \rightarrow F(\sigma)\right\}_{\sigma}
$$

will be called a weak $n$-cochain if for each morphism $\alpha: \sigma \rightarrow \tau$ in $\Sigma$, there

AMS (MOS) subject classifications (1970). Primary 55B05, 55G35; Secondary 54E45, $55 \mathrm{G} 45,55 \mathrm{~J} 20$.

Key words and phrases. Obstruction theory, shape, Čech type cohomology theory. 
is a cochain $g_{\alpha}: \partial C_{n}(\sigma) \rightarrow F(\tau)$ such that

$$
F(\alpha) f(\sigma)-f(\tau) C_{n}(\alpha)=g_{\alpha} \partial(\sigma) .
$$

Denoting by $\operatorname{Hom}_{f}\left(C_{n}, F\right)$ the abelian group of weak $n$-cochains from $C_{*}$ to $F$ and $\operatorname{Hom}_{f}\left(C_{*}, F\right)$ the corresponding cochain complex, we get cohomology groups

$$
H_{f}^{*}\left(C_{*} ; F\right)=H\left(\operatorname{Hom}_{f}\left(C_{*}, F\right)\right) .
$$

In particular, we are interested in the case where $X: \Sigma \rightarrow \mathrm{CW}_{*}$ is a diagram of $\mathrm{CW}$-complexes and $C_{*}=C_{*}(X)$, the corresponding diagram of cellular chain complexes. In this case we write $H_{f}^{*}(X ; F)$ instead of $H_{f}^{*}\left(C_{*}(X) ; F\right)$.

The cohomology groups related to a short exact sequence

$$
0 \rightarrow A_{*} \rightarrow B_{*} \rightarrow C_{*} \rightarrow 0
$$

of diagrams of chain complexes are connected by a long exact sequence, provided that the sequence is "locally split". There is also a universal coefficient theorem relating $H^{*}\left(C_{*} ; F\right)$ with the homology diagrams of $C_{*}, H_{*}\left(C_{*}\right)$, in $A b^{\Sigma}$.

(We adopt throughout the convention that the category of diagrams of type in a category $C$ is denoted by $C^{\Sigma}$.)

3. Local homotopy of weak maps. The corresponding notion in $\mathrm{CW}_{*}$ is as follows:

If $X, Y$ are objects in $\mathrm{CW}_{*}$ and $f=\{f(\sigma): X(\sigma) \rightarrow Y(\sigma)\}_{\sigma}$ is a family of maps in $\mathrm{CW}_{*}$ such that there is, for each $\alpha: \sigma \rightarrow \tau$ in $\Sigma$, a homotopy $H(\alpha): X(\sigma) \times I \rightarrow Y(\tau)$ from $Y(\alpha) f(\sigma)$ to $f(\tau) X(\alpha)$, then we say $f$ is a weak map of diagrams.

If $f$ and $g$ are weak maps from $X$ to $Y$, then they are locally homotopic if for each $\sigma$ in $\Sigma, f(\sigma)$ and $g(\sigma)$ are homotopic in $\mathrm{CW}_{*}$.

These notions of local homotopy and weak maps tie in, in the obvious way, with the cohomology theory of $\$ 2$ and, by using the homotopy pullbacks and pushouts of Peter Strain [6], we can construct a natural isomorphism

$$
[\quad ; K(F ; n)]_{f} \cong H_{f}^{n}(\quad ; F)
$$

where $K(F ; n)$ is an "Eilenberg-Mac Lane diagramme" for $F$ and $[X, Y]_{f}$ denotes the set of local homotopy class of weak maps from $X$ to $Y$.

The obstruction theory for weak maps now follows the classical pattern, as in Spanier [5]. There is a homotopy decomposition along the lines of the Moore-Postnikov construction, and analogues of the Hopf classification and extension theorems; however extension can only be guaranteed up to local homotopy. The primary obstructions for lifting a map pair, 


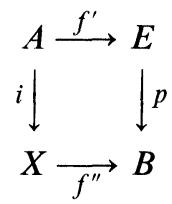

lie in the reduced cohomology group $\tilde{H}_{f}^{n}\left(X, A ; \pi_{n}(p)\right)$ where $\pi_{n}(p)$ is the first nonzero homotopy group-diagram of the map $p$. Secondary and higher obstruction can also be defined.

4. Obstructions in shape theory. If $X$ is a pointed compact metric space, one can form an inclusion inverse system $\boldsymbol{X}$ of pointed polyhedra with inverse limit $X$ (see for instance [2]). If $X$ and $Y$ are compact metric spaces and $\boldsymbol{X}$ and $\boldsymbol{Y}$ inclusion systems of polyhedra associated with them, a shape-map $f$ from $X$ to $Y$ is a functor $f: A_{Y} \rightarrow A_{X}$ between the indexing categories for $\boldsymbol{Y}$ and $\boldsymbol{X}$ respectively and a weak map $\boldsymbol{X}(f(\quad)) \stackrel{f}{\rightarrow} \boldsymbol{Y}(\quad)$. Hence the search for a diagonal to a commutative square

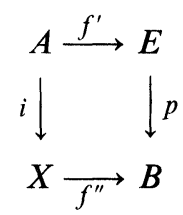

of compact metric spaces and shape maps reduces to looking for a diagonal for at least one of the squares

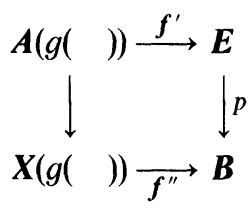

where $g$ is any functor from $A_{E}$ to $A_{X}$. (We can easily identify $A_{E}$ with $A_{B}$ as well as $A_{X}$ with $A_{A}$.) Thus the primary obstruction will lie in the limit group

$$
\bar{H}_{f}^{n}\left(X, A ; \pi_{n}(p)\right)=\frac{\lim }{\left(A_{E}, A_{X}\right)} \tilde{H}_{f}^{n}\left(X(g(\quad)), A(g(\quad)) ; \pi_{n}(p)\right)
$$

We thus get a series of obstruction sets

$$
O_{k}(f) \subset \bar{H}_{f}^{m_{s}}\left(X, A ; \pi_{m_{s}}(p)\right)
$$

where $O_{k}(f)$ is defined if and only if $0 \in O_{k-1}(f)$. We get as a simple application of this fact, that $A$ is a shape retract of $X$ in the sense of [1], if and only if $0 \in O_{k}(i)$ for all $k$ where $i: A \subset X$ is the inclusion. 
5. Conclusion. This theory is only in its infancy; no attempt has been made yet to calculate any obstructions and, for the sake of simplicity, all the usual classical conditions, such as $\pi_{1}(X)=\pi_{1}(A)=0$, have been assumed. However, it seems that this general method of working in a category of diagrams until the last minute, has paid off, even though the difficulties of the final situation, at present, dictate that the only application can be for compact metric spaces which have, of course, an extremely simple structure for their associated inverse systems. Thus the main difficulties left are the extension to compact Hausdorff spaces via shape theory, also to even more general spaces via Cech homotopy, and the problem of the calculation of the groups $\bar{H}_{f}^{n}(X, A ; F)$ and the obstructions. This latter problem will require a close analysis of the interrelationships between direct limits and spectral sequences.

\section{REFERENCES}

1. S. Mardešić, Retracts in shape theory, Glasnik Math. 6 (26) (1971), 153-163.

2. - A survey of the shape theory of compacta, III Prague Topology Sympos. (to appear).

3. T. Porter, Čech homotopy. I, II, J. London Math. Soc. (2) 6 (1973), 429-436, 667-675.

4. - Borsuk's theory of shape and Čech homotopy, Math. Scand. (to appear).

5. E. H. Spanier, Algebraic topology, McGraw-Hill, New York, 1966. MR 35 \# 1007.

6. P. Strain, Categories for abstract homotopy theory, Thesis, Cambridge, England, 1972.

Department of Mathematics, University College, Cork, Eire 